\title{
43. Urinary Amino Acids of Hyperthyroid Patient
}

\author{
By Yoshiya SonodA \\ Department of Biochemistry, Okayama University Medical School, Okayama \\ (Comm. by T. ShimizU, M.J.A., March 12, 1957)
}

In a previous paper, ${ }^{1)}$ it has been reported that several ninhydrinpositive ampholytes, which are stable to acid hydrolysis and give the unusual large spots on paper chromatogram, are found to be excreted in urine of a hyperthyroid patient. Among the ampholytes mentioned above sarcosine and $\beta$-alanine have already been identified on paper chromatogram by using a known sample of each.

Subsequent study ${ }^{2}$ has revealed that thyroxine treatment has led to the increased excretion of lysine, sarcosine, $\beta$-alanine, and an unidentified ampholyte in rabbit urine.

This communication is concerned with further identification of three unidentified amino acids excreted by a hyperthyroid patient. Now it seems quite probable that these amino acids are pipecolic acid, $\delta$-aminovaleric acid, and $\beta$-aminoisobutyric acid.

Experimental. Methods. As described previously, ${ }^{3)}$ urine desalted by the method of Carsten ${ }^{4)}$ was applied on a filter paper, and $n$-butanolacetic acid-water $(5: 1.2: 5 \mathrm{v} / \mathrm{v})$ and phenol-water $\left(4: 1 \mathrm{v} / \mathrm{v}, 0.3 \% \mathrm{NH}_{3}\right.$ in the chamber) were used as the solvents for two-way chromatography. $0.1 \%$ ninhydrin in water-saturated $n$-butanol was used for color development.

Materials. $\beta$-Aminoisobutyric acid, $\alpha$-aminobutyric acid, and $\gamma$ aminobutyric acid were kindly furnished by Prof. Dr. K. Ichihara, and pipecolic acid was a generous gift from Prof. Dr. M. Suda. $\delta$ Aminovaleric acid was synthesized by a modification of Wallach's method. ${ }^{5)}$

The other amino acids tested were commercial products.

Results. Fig. 1 shows the urinary amino acid pattern of a hyperthyroid patient. Spots (a) and (b) were previously identified to be $\beta$ alanine and sarcosine respectively. ${ }^{1)}$ Urinary serine is usually contained in fairly large amounts. But in this patient, serine almost completely disappeared from the urine while sarcosine increased. This fact has agreed with the observation that sarcosine oxidase activity and serine formation in rat liver are reduced when the rat is in a hyperthyroid state. ${ }^{6)}$

When $\beta$-aminoisobutyric acid and pipecolic acid were spotted on a zero point with the urine sample of the patient, these amino acids have just overlapped on the spot (c) and (e) respectively as shown in 
Fig. 2. Referring to the report of Morrison, ${ }^{7)}$ pipecolic acid always has a little higher Rf value than proline in any solvents so far tested. This fact would indicate that the spot (e) is pipecolic acid.

Similary, $\delta$-aminovaleric acid has just overlapped on the spot (d) (Fig. 3). From the Rf values of $\omega$-amino acids reported by Synge, ${ }^{8)}$ spot (d) may also be considered to be $\delta$-aminovaleric acid. As reported previously, ${ }^{2}$ spot (d) has also been found in rabbit urine previously

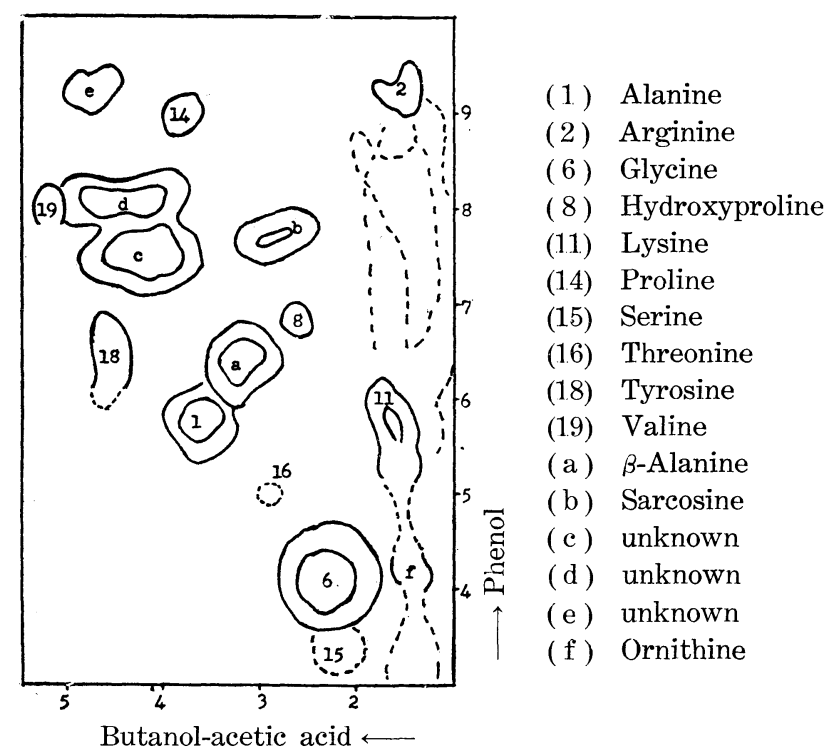

Fig. 1. Urinary amino acids of hyperthyroid patient

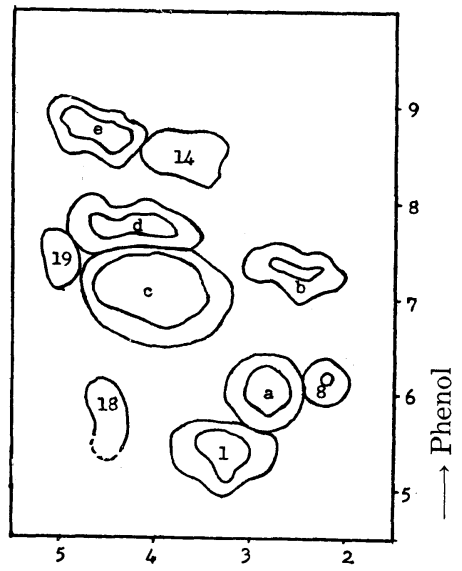

Butanol-acetic acid

Fig. 2. Urinary amino acids of hyperthyroid patient + pipecolic acid (e)

$+\beta$-aminoisobutyric acid (c)

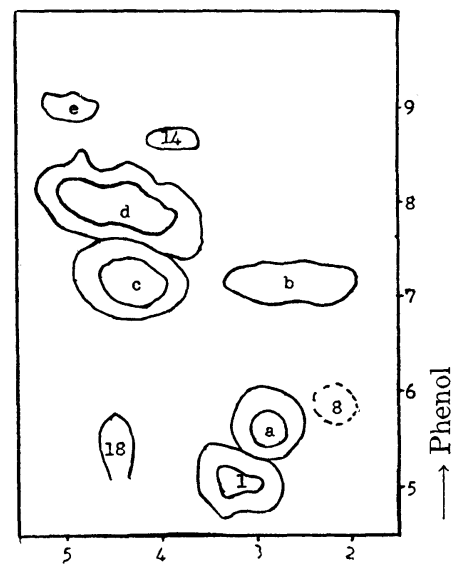

Butanol-acetic acid $\longleftarrow$

Fig. 3. Urinary amino acids of hyperthyroid patient $+\hat{\delta}$-aminovaleric acid $(\mathrm{e})$ 
treated with thyroxine, so $\delta$-aminovaleric acid can be considered to be one of the specific amino acids excreted by animals when in a hyperthyroid state.

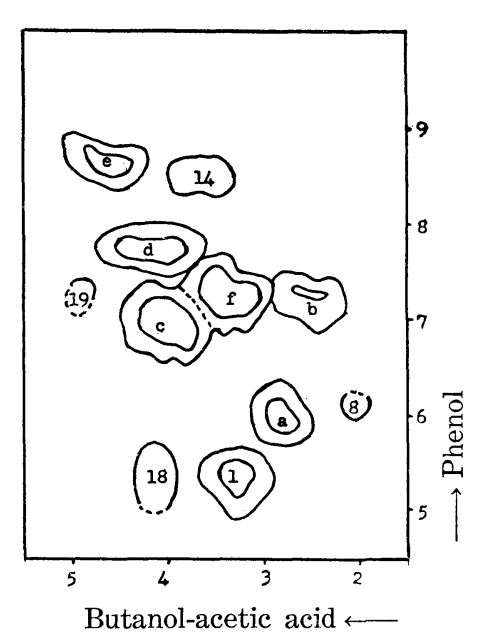

Fig. 4. Urinary amino acids of hyperthyroid patient + pipecolic acid (e) $+\gamma$-aminobutyric acid (f)

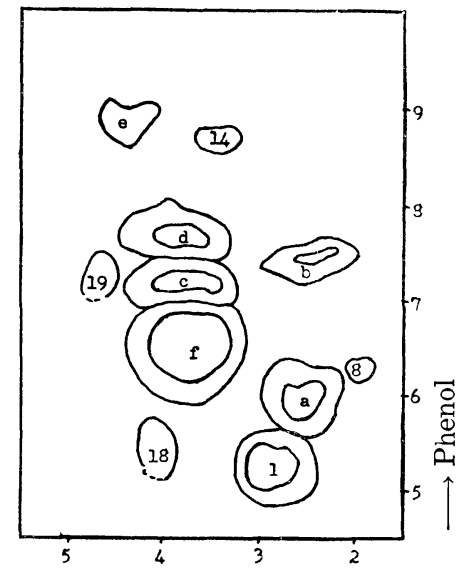

Butanol-acetic acid $\longleftarrow$

Fig. 5. Urinary amino acids of hyperthyroid patient $+\alpha$-aminobutyric acid (f)

As shown in Fig. 4, pipecolic acid has again overlapped on the spot (e), but $\gamma$-aminobutyric acid has given a separate spot (f).

$\alpha$-Aminobutyric acid has never overlapped with either spot (c) or (d) (Fig. 5).

Discussion. Among the basic amino acids excreted in the urine of the hyperthyroid patient under examination, lysine was the most abundant. Pipecolic acid is known to be a normal metabolite of lysine $^{9)}$ and has never been found in any human urines tested. ${ }^{10)}{ }^{12}$ ) $\delta$-Aminovaleric acid which has also never been found in any human urines tested may be derived from $\alpha$-keto analogues of lysine ( $\alpha$-keto$\varepsilon$-amino caproic acid), though $\delta$-aminovaleric acid has not been considered to be a normal metabolite of lysine. ${ }^{13)}$ It is well known that, when tracing a two dimensional chromatogram of series of $\omega$-amino acids, these spots fall on a smooth curve. This relation is also observed between spots (6), (a), (f), and (d) on Fig. 1 and Fig. 4. This fact also indicates that spot (d) is $\delta$-aminovaleric acid. Furthermore, the latest observation (unpublished data) shows that lysine, $\alpha$-aminoadipic acid, and $\beta$-alanine are found to be increased in the extractive substances of a rabbit muscle treated with thyroxine. From these considerations, it may be suggested that thyroid hormone may have an important effect on lysine metabolism. As compared to a normal man the $\beta$ aminoisobutyric acid had increased in this hyperthyroid patient, how- 
ever, fairly large amounts of this amino acid are usually found in normal Japanese urines, and this ampholyte did not increase in the rabbit urine treated with thyroxine. So at present it is not sure whether this amino acid has some relation with the thyroid function or not. Further confirmations of these unusual amino acids found in the urine of a hyperthyroid patient are now being made.

Summary. Several unusual amino acids were found to be increased in a hyperthyroid patient. They are quite probably pipecolic acid, $\delta$-aminovaleric acid, lysine, $\beta$-alanine, $\beta$-aminoisobutyric acid, and sarcosine.

The thyroid gland may have an important effect on lysine metabolism.

Grateful acknowledgment is expressed to Professor S. Mizuhara for his interest in this work. This investigation was supported by a Grant for Scientific Research of the Ministry of Education.

\section{References}

1) Sonoda, Y.,: J. Jap. Biochem. Soc. (1957) (in press).

2) Sonoda, Y.,: J. Jap. Biochem. Soc. (1957) (in press).

3) Kurahashi, K., Nakamura, R., and Sonoda, Y.,: J. Jap. Biochem. Soc. (1957) (in press).

4) Carsten, M. E.,: J. Am. Chem. Soc., 74, 5954 (1952).

5) Wallach, O.,: Ann. Chem., 312, 179 (1900).

6) Swendseid, M. E., Swarson, A. L., and Bethell, F. H.,: Arch. Biochem., 41, 138 (1952).

7) Morrison, R. I.,: Biochem. J., 50, XIV (1952); ibid., 53, 474 (1953).

8) Synge, R. L. M.,: Bicchem. J., 48, 429 (1951).

9) Rəthstein, M., and Miller, L. L.,: J. Am. Chem. Sэc., 75, 4731. (1953); J. Am. Chem. Səc., 76, 1459 (1.954).

10) Nakamura, R.,: J. Jap. Bicchem. Sxc. (1957) (in press).

11) Harris, H.,: “Aminoaciduria" in Rapports 3ème Congrès International de Biochimie, Bruxelles, 34 (1955).

12) Westall, R. G.,: Biochem. J., 60, 247 (1955).

13) Rothstein, M., and Miller, L. L.,: J. Biol. Chem., 206, 243 (1954). 